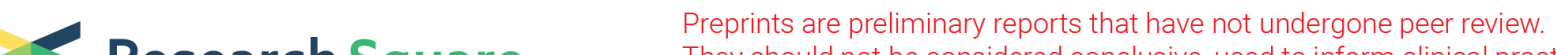 Research Square They should not be considered conclusive, used to inform clinical practice, or referenced by the media as validated information.
}

\section{A Retrospective Study of Pegylated Liposomal Doxorubicin Combined with S-1 and Low-dose Oxaliplatin as First-Line Treatment for Advanced Gastric Cancer}

\section{Jian Ma}

Changzhou Tumor Hospital Affiliated to Soochow University

\section{Quanliang Yang}

Changzhou Tumor Hospital Affiliated to Soochow University

\section{Yanzhi Bi}

Changzhou Tumor Hospital Affiliated to Soochow University

\section{Min Xiao}

Changzhou Tumor Hospital Affiliated to Soochow University

\section{Xiaoqian Li}

Changzhou Tumor Hospital Affiliated to Soochow University

\section{Qiu Zhao}

Changzhou Tumor Hospital Affiliated to Soochow University

\section{Wei Ye}

Changzhou Tumor Hospital Affiliated to Soochow University

\section{Wenjing Ji}

Changzhou Tumor Hospital Affiliated to Soochow University

\section{Yang Ling ( $\sim$ lingyang2015@aliyun.com )}

Changzhou Cancer Hospital of Soochow University

\section{Research article}

Keywords: pegylated liposomal doxorubicin, advanced gastric cancer, SOX

Posted Date: October 26th, 2020

DOl: https://doi.org/10.21203/rs.3.rs-95658/v1

License: (c) (1) This work is licensed under a Creative Commons Attribution 4.0 International License. Read Full License 


\section{Abstract}

Background: At present, the first-line treatment of advanced gastric cancer is oxaliplatin combined with fluorouracil (capecitabine or S-1). Is there a three-drug regimen in which the curative effect is higher than that of the two-drug regimen, but the side effects are not significantly increased? This retrospective study evaluated the efficacy and side effects of pegylated liposomal doxorubicin (PLD) combined with S-1 and low-dose oxaliplatin as first-line treatment for advanced gastric cancer.

Methods: Advanced gastric cancer patients who received PLD combined with S-1 and low-dose oxaliplatin (D-SOX) or standard SOX as first-line chemotherapy from Jan 2015 to Dec 2018 were included in the study. Overall survival (OS), progression-free survival (PFS), response rate (RR), and safety were assessed.

Results: A total of 52 patients were included in this study. There were 23 cases in D-SOX group and 29 cases in SOX group. The median PFS was 7.1 months (95\% Cl 5.66-8.54 months) in the SOX group and 9.1 months ( $95 \% \mathrm{Cl} 4.2-14.0$ months) in the $\mathrm{D}-\mathrm{SOX}$ group $(\mathrm{P}=0.036)$. The median OS was 12.5 months (95\% Cl 7.31-17.70 months) in the SOX group and 19.5 months (95\% $\mathrm{Cl} 16.23-22.7$ months) in the DSOX group $(P=0.017)$. The incidence of treatment-related grade III-IV adverse events was no more than $10 \%$. The incidence of neurotoxicity was significantly reduced in the D-SOX group.

Conclusions: This study suggests that PLD combined with S-1 and low-dose oxaliplatin might be a safe and more effective treatment for advanced gastric cancer.

\section{Introduction}

Gastric cancer was the fifth most common diagnosed cancer type in 2018 and was responsible for $8.2 \%$ of all deaths from cancer in 2018[1]. It is the third most common cause of cancer-related death after lung and colorectal cancer.

Some patients have metastasis at the time of diagnosis, lose the opportunity of operation, and can only receive chemotherapy, radiotherapy, and other treatments. In terms of chemotherapy, fluorouracil (capecitabine, S-1, etc.), paclitaxel, and platinum are the most common used drugs. NCCN guidelines recommend two-drug regimens (fluorouracil + platinum). In Asia, S-1 is mostly used, while in North America, capecitabine is widely used, both of which have been proved to be equivalent in efficacy[2]. Because of the large side effects, the three-drug regimen is only reserved for patients in good condition and can often evaluate the toxic reaction. Anthracycline antibiotics, once the first choice for perioperative chemotherapy for gastric cancer[3], are also used in chemotherapy for advanced gastric cancer[4]. However, due to the unique side effects of anthracycline antibiotics and the development of clinical trials of taxanes, the application of anthracycline antibiotics is greatly restricted. At present, the recommended level of anthracycline antibiotics in NCCN chemotherapy for advanced gastric cancer is only 2B. 
With the emergence of pegylated liposome doxorubicin, its unique mechanism reduces the side effects and enhances the targeting of drugs, but its application in gastric cancer is rarely reported. In the past two decades, there are only two small sample phase II clinical trials[5, 6]. The most recent one has been nearly ten years ago, so how effective is it in combination with fluorouracil preparations that are commonly used now? In this paper, we analyzed the data of patients who had used PLD combined with S-1 and lowdose oxaliplatin in the past few years, and compared it with the standard SOX regimen for the same period.

\section{Materials And Methods Patients}

Advanced gastric cancer (AGC) patients who received SOX or PLD combined with S-1 and low-dose oxaliplatin as first-line chemotherapy in our institution from 2015-2018 were included in the study. The selection criteria were as follows: 20 years later; Eastern Cooperative Oncology Group (ECOG) performance status of 2 or less. They were evaluated by clinicians that they can tolerate chemotherapy. Pathologically confirmed locally advanced non-resectable or metastatic gastric cancer and HER-2 negative. No chemotherapy and radiotherapy after diagnosis $\square$ adjuvant chemotherapy was allowed if

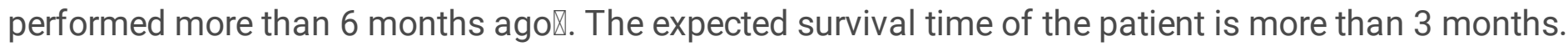
No less than two cycles of chemotherapy.

\section{Treatment}

The patients in the SOX regimen group received oxaliplatin $130 \mathrm{mg} / \mathrm{m}^{2} \mathrm{~d} 1$, S- $140 \mathrm{mg} / \mathrm{m}^{2}$ bid d1-14; DSOX regimen: PLD $25 \mathrm{mg} / \mathrm{m}^{2}$, oxaliplatin $85 \mathrm{mg} / \mathrm{m}^{2}, \mathrm{~S}-140 \mathrm{mg} / \mathrm{m}^{2}$ bid d1-14, all of which had a 21-day cycle. The adjustment of the chemotherapy dose, the postponement of the cycle was decided by the doctors, and the treatment continues until the disease progresses or has unacceptable side effects, or the patient gives up the treatment.

\section{Assessments}

This retrospective analysis was conducted to compare the efficacy and safety of D-SOX regimens and SOX regimens, including ORR, PFS, OS. The time of disease progression of some patients is unknown, so the PFS data is missing, only the OS data are included. The curative effect was evaluated with enhanced $\mathrm{CT}$, according to RECIST 1.1. The security analysis is based on National Cancer Institute Common Terminology Criteria for Adverse Events (CTCAE) ver. 5.0.

This study was approved by the Ethical Committee of Changzhou Cancer Hospital of Soochow University.

\section{Statistical analysis}

There are two groups: SOX group and D-SOX group. T-test and chi-square tests were used to compare the baselines of patients. The OS was defined as the time from initiation of chemotherapy to the date of death from any cause or censored at the latest follow-up for surviving patients. PFS was defined as the 
time from initiation of chemotherapy to the date of disease progression or death, and survivors without disease progression were censored at the last contact. OS and PFS curves were estimated using the Kaplan-Meier method. The hazard ratio (HR) was estimated using the Cox proportional hazards model. Univariate and multivariable analyses were also performed using the Cox proportional hazards model. Univariate analysis was performed to explore prognostic factors $\square$ Statistical analyses were performed using the SPSS 26.

\section{Results}

\section{Patients}

According to the inclusion criteria, a total of 52 patients were selected, including 29 patients in SOX group and 23 patients in D-SOX group. The patient characteristics of both groups are shown in Table 1. The baseline characteristics were generally well balanced between the two groups.

\section{Efficacy and Safety}

There were 29 cases in the SOX group. The curative effect could be evaluated in 22 cases and could not be evaluated or no further evaluation in 7 cases. ORR $27.6 \%$, DCR $69 \%$. In the D-SOX group, the curative effect could be evaluated in 16 cases and could not be evaluated or no further evaluation in 7 cases. ORR $30.4 \%$, DCR 69.5\% (shown in Table 2).

The median PFS was 7.1 months (95\% Cl 5.66-8.54 months) in the SOX group and 9.1 months ( $95 \% \mathrm{Cl}$ 4.2-14.0 months) in the $D-S O X$ group $(P=0.036)$ (shown in Fig. 1$)$. The median OS was 12.5 months (95\% Cl 7.31-17.70 months) in the SOX group and 19.5 months (95\% $\mathrm{Cl} 16.23-22.7$ months) in the DSOX group $(P=0.017)$ (shown in Fig. 2). Univariate analysis showed an association between OS and abdominal and retroperitoneal lymph node metastasis $(p=0.010) \square$ No. of involved organs $(p=0.004) \square$ therapy regimens $(P=0.019)$;Multivariate analysis of variance suggested that OS was related to chemotherapy regimen $(H R=0.467, \mathrm{P}=0.045,95 \% \mathrm{Cl} 0.222-0.982)$ (shown in Table 3).

The adverse events (AEs) of all patients are summarized in Table 4. Most patients can tolerate chemotherapy, and the addition of PLD did not significantly increase the incidence of side effects. Among them, gastrointestinal reaction and neutropenia are more common, but the incidence of grade 1-2 is higher, and the incidence of grade 3-4 is lower. Secondly, the incidence of thrombocytopenia was slightly higher. It is worth noting that the incidence of neurotoxicity in the D-SOX group was significantly reduced due to the use of lower doses of oxaliplatin. No obvious cardiotoxicity was observed in D-SOX.

\section{Discussion}

PLD has its unique mechanism[7-10]: 1, low immunogenicity, little side effects, phospholipid molecules are amphiphilic molecules, improve solubility; 2 , almost $100 \%$ of liposomes exist in the form of liposomes in the blood circulation; 3 , after liposomes are biodegraded, the drug is released slowly, and the circulation 
time of the drug in the body $\left(T_{1 / 2} 55-70 \mathrm{~h}\right) ; 4$, reasonable liposome size-the average particle size is about $90 \mathrm{~nm}$. 5.EPR effect, tumor vascular permeability increases, and liposomes are smaller, which can pass through defective blood vessels, thus increasing the local drug concentration in tumor tissue about 20 times that of common doxorubicin.

The toxicity and side effects, especially cardiotoxicity, have been significantly reduced, which may be due to the low peak concentration of free doxorubicin in plasma after administration of PLD and the trend of accumulation of liposome drugs in the myocardium[8]. It was not until a significantly higher lifetime dose was reached that the cardiotoxicity of the nanoparticle preparation PLD was observed[11, 12]. However, Sarah E. Gill [13] pointed out that PLD has no obvious cardiotoxicity, which is proved by the stability of LVEF even after high cumulative dose treatment. In the absence of other risk factors, routine monitoring of LVEF does not seem to be necessary or cost-effective.

However, PLD has its unique side effects, especially mucositis and palmar-plantar erythrodysesthesia (PPE). Besides, some patients may have hypersensitivity reaction(HSR), that is, acute hypersensitivity (infusion) reactions referred to as complement activation-related pseudoallergy (CARPA)[14-16], To minimize HSR to PLD, an initial reduced rate of infusion of $0.1-0.2 \mathrm{mg} / \mathrm{min}$ of PLD is warranted. In the event of HSR, alternative therapy must be privileged, and if necessary, careful rechallenge with PLD may be attempted, however, relapse of HSR may occur[17].

The tolerance of the drug has been increased, and the mechanism of action has been improved. what about the curative effect? At present, there is no clear evidence that PLD has a better curative effect than traditional doxorubicin. Meta-analysis showed [18] that in the case of ovarian cancer, the PFS benefit of the PLD-based regimen was significant, although the OS did not improve. Another meta-analysis[19] showed that the efficacy of liposome (liposome anthracyclines, liposome cisplatin, etc.) was not different from that of conventional chemotherapy, and even the subgroup of liposome doxorubicin did not show an obvious advantage. At the same time, this article conducted a meta-analysis of 11 preclinical studies of liposome anthracyclines. In contrast to the clinical results, animal studies showed that the survival rate of mice treated with PLD was significantly higher than that of traditional anthracyclines (HR0.39; $95 \% \mathrm{Cl}$ 0.27-0.56). It seems that the potential of PLD has not been fully exploited.

At present, it has been widely used in hematological tumors, ovarian cancer, and breast cancer[12, 2023]. However, PLD has not been widely used in the treatment of gastric cancer. A phase II clinical trial in 2001[24] suggested that liposome doxorubicin alone had no significant antineoplastic activity in gastric cancer. Recchia[25] et al used PLD combined with oxaliplatin in the salvage treatment of gastric cancer, median PFS 5.8 months, OS 9.2 months. In 2005, the phase II clinical trial of Gnad-Vogt et al[5] proved that PLD combined with mitomycin and fluorouracil achieved a response rate of $47 \%$ in the first-line treatment of gastric cancer, with a median PFS of 8.4 months and an OS 14.7 months. In 2011, Cascinu et al[6] conducted a phase II clinical trial in which PLD, cisplatin, and 5-FU were used in the first-line treatment of gastric cancer, compared with mitomycin C, cisplatin, and 5-Fu. ORR, TPP, and OS were superior to the control group. This trial was once cited by NCCN guidelines and later deleted. In the past 
nine years, there has been no further clinical study, and the treatment of gastric cancer has been developed. At present, XELOX or SOX regimen is the first choice, while the DCF regimen is recommended in NCCN guidelines for patients with the good general condition and frequent monitoring of side effects. Thus, is there a regimen that is safe and more effective than XELOX or SOX?

In the D-SOX group,we reduced the dose of oxaliplatin regimen from $130 \mathrm{mg} / \mathrm{m}^{2}$ to $85 \mathrm{mg} / \mathrm{m}^{2}$, plus pegylated liposome doxorubicin, $25 \mathrm{mg} / \mathrm{m}^{2}$, thus reducing the cumulative dose of oxaliplatin, thus reducing the possibility of peripheral neurotoxicity. At the same time, the cumulative dose of PLD, even after six standard cycles of chemotherapy, was only $150 \mathrm{mg} / \mathrm{m}^{2}$. At this dose level, no serious adverse reactions were observed, including cardiotoxicity, mucositis, etc., and the incidence of alopecia was rare. There are statistical differences in PFS and OS between the two groups, the root cause of which lies in the chemotherapy regimen, but it may also be related to the better economic conditions of patients in the D-SOX group and better palliative treatment (PLD is not included in medical insurance). In previous years, targeted therapy was not widely used, and only 2 cases in each group received targeted therapy. There was no significant difference in ORR and DCR between the two groups, which was related to the small sample size and the termination of treatment without evaluating the curative effect of some patients. On the other hand, the lack of follow-up treatment also reduces the impact of differences in follow-up treatment on OS.

With the development of molecular targeted therapy and immunotherapy, there is a significant decrease in the number of patients with chemotherapy exceeding the second line in clinical practice, so the use of as many effective drugs as possible in first-line and second-line therapy may benefit patients to the greatest extent. Of course, side effects must be considered. Therefore, the low-dose combination of the three drugs may be one of the options.

Of course, this article is only a retrospective study and the number of cases is relatively small. The selection of cases does not strictly follow the principle of randomization, so there may be bias. It is hoped that there will be well-designed clinical trials to verify the effectiveness of the D-SOX regimen in the future.

In conclusion, pegylated liposomal doxorubicin combined with S-1 and low-dose oxaliplatin might be a safe and more effective treatment for advanced gastric cancer

\section{Abbreviations}

AGC: advanced gastric cancer

PLD: pegylated liposomal doxorubicin

OS: overall survival

PFS: progression-free survival 
ORR: Objective response rate

PPE: palmar-plantar erythrodysesthesia

HSR: hypersensitivity reaction

CARPA: complement activation-related pseudoallergy

\section{Declarations}

\section{Acknowledgments}

We thank the staff of the department of Oncology of Changzhou Cancer Hospital of Soochow University.

\section{Funding}

This study was supported by Changzhou Sci\&Tech Program, China (Grant No.: CJ20160049).

\section{Availability of data and materials}

The datasets used and/or analyzed during the current study are available from the corresponding author on reasonable request.

\section{Authors' contributions}

$J M, Q Y$, and $Y L$ designed the study; JM, QY, YB acquired the data; JM analyzed and interpreted the data and drafted the manuscript. All authors have read and approved the manuscript.

\section{Competing interests}

The authors declare that they have no competing interests.

\section{Consent for publication}

Written informed consent was obtained from the patient for publication of this case report and any accompanying images. A copy of the written consent is available for review by the Editor of this journal.

\section{Ethics approval and consent to participate}

This study was approved by the Ethical Committee of Changzhou Cancer Hospital of Soochow University.

\section{References}

1. Bray F, Ferlay J, Soerjomataram I, Siegel RL, Torre LA, Jemal A: Global cancer statistics 2018: GLOBOCAN estimates of incidence and mortality worldwide for 36 cancers in 185 countries. $C A$ Cancer J Clin 2018, 68(6):394-424. 
2. Kim GM, Jeung HC, Rha SY, Kim HS, Jung I, Nam BH, Lee KH, Chung HC: A randomized phase II trial of S-1-oxaliplatin versus capecitabine-oxaliplatin in advanced gastric cancer. Eur J Cancer 2012, 48(4):518-526.

3. Cunningham D, Allum Wh Fau - Stenning SP, Stenning Sp Fau - Thompson JN, Thompson Jn Fau Van de Velde CJH, Van de Velde Cj Fau - Nicolson M, Nicolson M Fau - Scarffe JH, Scarffe Jh Fau Lofts FJ, Lofts Fj Fau - Falk SJ, Falk Sj Fau - Iveson TJ, Iveson Tj Fau - Smith DB et al: Perioperative chemotherapy versus surgery alone for resectable gastroesophageal cancer. 2006(1533-4406 (Electronic)).

4. Cunningham D, Starling N Fau - Rao S, Rao S Fau - Iveson T, Iveson T Fau - Nicolson M, Nicolson M Fau - Coxon F, Coxon F Fau - Middleton G, Middleton G Fau - Daniel F, Daniel F Fau - Oates J, Oates J Fau - Norman AR, Norman AR: Capecitabine and oxaliplatin for advanced esophagogastric cancer. 2008(1533-4406 (Electronic)).

5. Gnad-Vogt SU, Hofheinz RD, Saussele S, Kreil S, Willer A, Willeke F, Pilz L, Hehlmann R, Hochhaus A: Pegylated liposomal doxorubicin and mitomycin $\mathrm{C}$ in combination with infusional 5-fluorouracil and sodium folinic acid in the treatment of advanced gastric cancer: results of a phase II trial. AntiCancer Drugs 2005, 16(4):435-440.

6. Cascinu S, Galizia E, Labianca R, Ferrau F, Pucci F, Silva RR, Luppi G, Beretta GD, Berardi R, Scartozzi $\mathrm{M}$ : Pegylated liposomal doxorubicin, 5-fluorouracil and cisplatin versus mitomycin-C, 5-fluorouracil and cisplatin for advanced gastric cancer: a randomized phase II trial. Cancer Chemother Pharmacol 2011, 68(1):37-43.

7. Sadzuka Y, Sugiyama I, Tsuruda T, Sonobe T: Characterization and cytotoxicity of mixed polyethyleneglycol modified liposomes containing doxorubicin. Int J Pharm 2006, 312(1-2):83-89.

8. Gabizon A, Martin F: Polyethylene glycol-coated (pegylated) liposomal doxorubicin. Rationale for use in solid tumours. (0012-6667 (Print)).

9. Caponigro F, Comella P Fau - Budillon A, Budillon A Fau - Bryce J, Bryce J Fau - Avallone A, Avallone A Fau - De Rosa V, De Rosa V Fau - Ionna F, Ionna F Fau - Comella G, Comella G: Phase I study of Caelyx (doxorubicin HCL, pegylated liposomal) in recurrent or metastatic head and neck cancer. (0923-7534 (Print)).

10. Lyseng-Williamson KA, Duggan St Fau - Keating GM, Keating GM: Pegylated liposomal doxorubicin: a guide to its use in various malignancies. 2013(1179-190X (Electronic)).

11. Uyar D, Kulp B, Peterson G, Zanotti K, Markman M, Belinson J: Cardiac safety profile of prolonged (>or=6 cycles) pegylated liposomal doxorubicin administration in patients with gynecologic malignancies. Gynecol Oncol 2004, 94(1):147-151.

12. O'Brien ME, Wigler N, Inbar M, Rosso R, Grischke E, Santoro A, Catane R, Kieback DG, Tomczak P, Ackland SP et al: Reduced cardiotoxicity and comparable efficacy in a phase III trial of pegylated liposomal doxorubicin $\mathrm{HCl}$ (CAELYX/Doxil) versus conventional doxorubicin for first-line treatment of metastatic breast cancer. Ann Oncol 2004, 15(3):440-449. 
13. Gill SE, Savage K, Wysham WZ, Blackhurst DW, Winter WE, Puls LE: Continuing routine cardiac surveillance in long-term use of pegylated liposomal doxorubicin: is it necessary? Gynecol Oncol 2013, 129(3):544-547.

14. Szebeni J, Muggia F, Gabizon A, Barenholz Y: Activation of complement by therapeutic liposomes and other lipid excipient-based therapeutic products: Prediction and prevention. Adv Drug Del Rev 2011, 63(12):1020-1030.

15. Szebeni J: Complement activation-related pseudoallergy: A stress reaction in blood triggered by nanomedicines and biologicals. Mol Immunol 2014, 61(2):163-173.

16. Szebeni J, Storm G: Complement activation as a bioequivalence issue relevant to the development of generic liposomes and other nanoparticulate drugs. Biochem Biophys Res Commun 2015, 468(3):490-497.

17. Ingen-Housz-Oro S, Pham-Ledard A, Brice P, Lebrun-Vignes B, Zehou O, Reitter D, Ram-Wolff C, Dupin $\mathrm{N}$, Bagot $\mathrm{M}$, Chosidow $\mathrm{O}$ et al: Immediate hypersensitivity reaction to pegylated liposomal doxorubicin: management and outcome in four patients. Eur $J$ Dermato/ 2017, 27(3):271-274.

18. Staropoli N, Ciliberto D, Botta C, Fiorillo L, Grimaldi A, Lama S, Caraglia M, Salvino A, Tassone P, Tagliaferri P: Pegylated liposomal doxorubicin in the management of ovarian cancer. Cancer Biol Ther 2014, 15(6):707-720.

19. Petersen GH, Alzghari SK, Chee W, Sankari SS, La-Beck NM: Meta-analysis of clinical and preclinical studies comparing the anticancer efficacy of liposomal versus conventional non-liposomal doxorubicin. J Controlled Release 2016, 232:255-264.

20. Orlowski RZ, Nagler A, Sonneveld P, Blade J, Hajek R, Spencer A, San Miguel J, Robak T, Dmoszynska A, Horvath $\mathrm{N}$ et al: Randomized phase III study of pegylated liposomal doxorubicin plus bortezomib compared with bortezomib alone in relapsed or refractory multiple myeloma: combination therapy improves time to progression. J Clin Oncol 2007, 25(25):3892-3901.

21. Pujade-Lauraine E, Wagner U, Aavall-Lundqvist E, Gebski V, Heywood M, Vasey PA, Volgger B, Vergote I, Pignata S, Ferrero A et al: Pegylated Liposomal Doxorubicin and Carboplatin Compared With Paclitaxel and Carboplatin for Patients With Platinum-Sensitive Ovarian Cancer in Late Relapse. $J$ Clin Oncol 2010, 28(20):3323-3329.

22. Mutch DG, Orlando M, Goss T, Teneriello MG, Gordon AN, McMeekin SD, Wang Y, Scribner DR, Jr., Marciniack M, Naumann RW et al: Randomized phase III trial of gemcitabine compared with pegylated liposomal doxorubicin in patients with platinum-resistant ovarian cancer. $\mathrm{J}$ Clin Oncol 2007, 25(19):2811-2818.

23. Gordon AN, Tonda M, Sun S, Rackoff W, Doxil Study I: Long-term survival advantage for women treated with pegylated liposomal doxorubicin compared with topotecan in a phase 3 randomized study of recurrent and refractory epithelial ovarian cancer. Gynecol Oncol 2004, 95(1):1-8.

24. Thomas A, O'Byrne K, Furber L, Jeffery K, Steward W: A phase II study of Caelyx, liposomal doxorubicin: lack of activity in patients with advanced gastric cancer. Cancer Chemother Pharmacol 2001, 48(4):266-268. 
25. Recchia F, Candeloro G, Guerriero G, Piazze J, Desideri G, Necozione S, Rea S: Liposomal pegylated doxorubicin and oxaliplatin as salvage chemotherapy in patients with metastatic gastric cancer treated earlier. Anti-Cancer Drugs 2010, 21(5):559-564.

\section{Tables}

Due to technical limitations, table PDF is only available as a download in the Supplemental Files section.

\section{Figures}

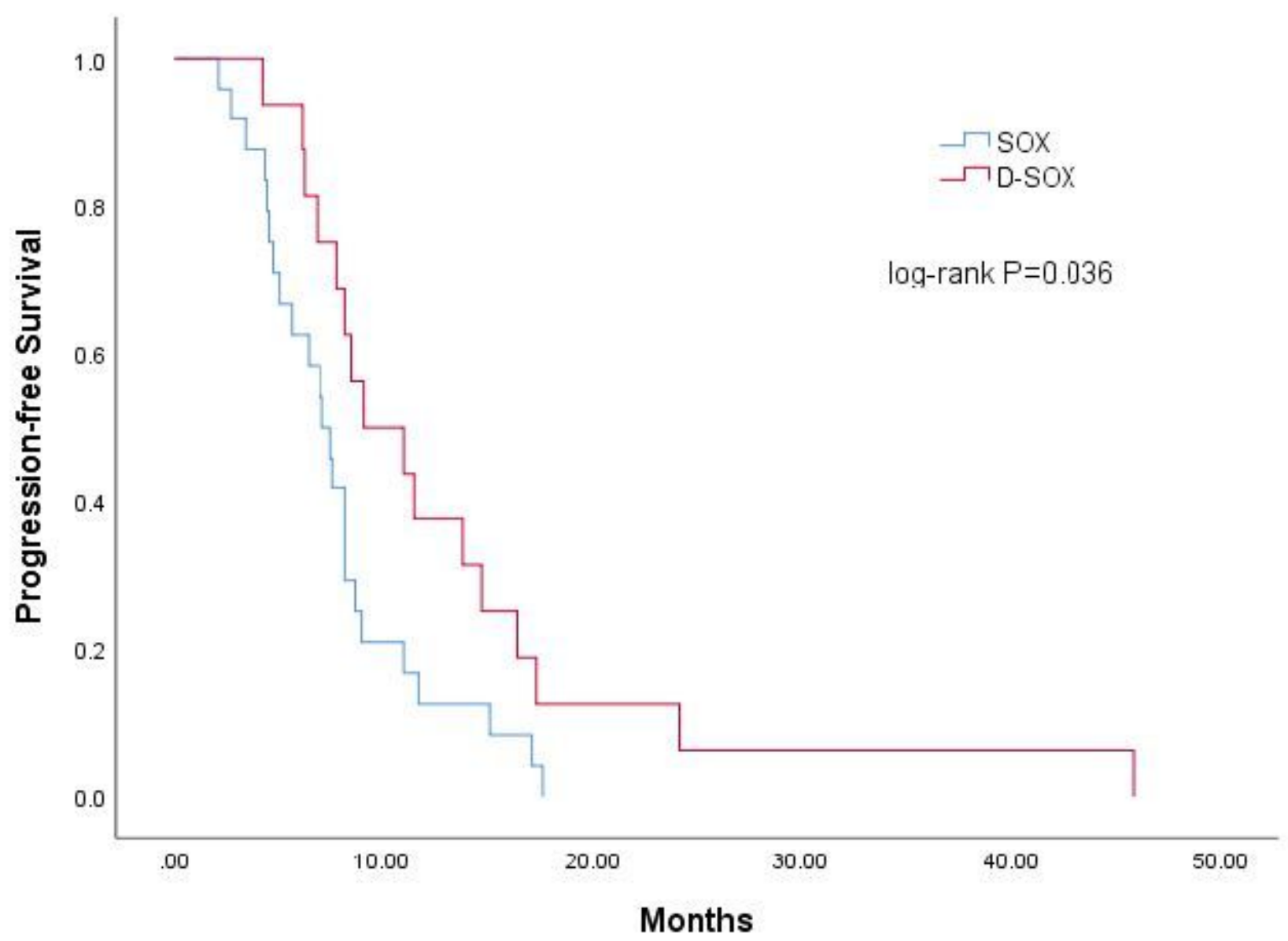

Figure 1

Kaplan-Meier progression-free survival for D-SOX versus SOX 


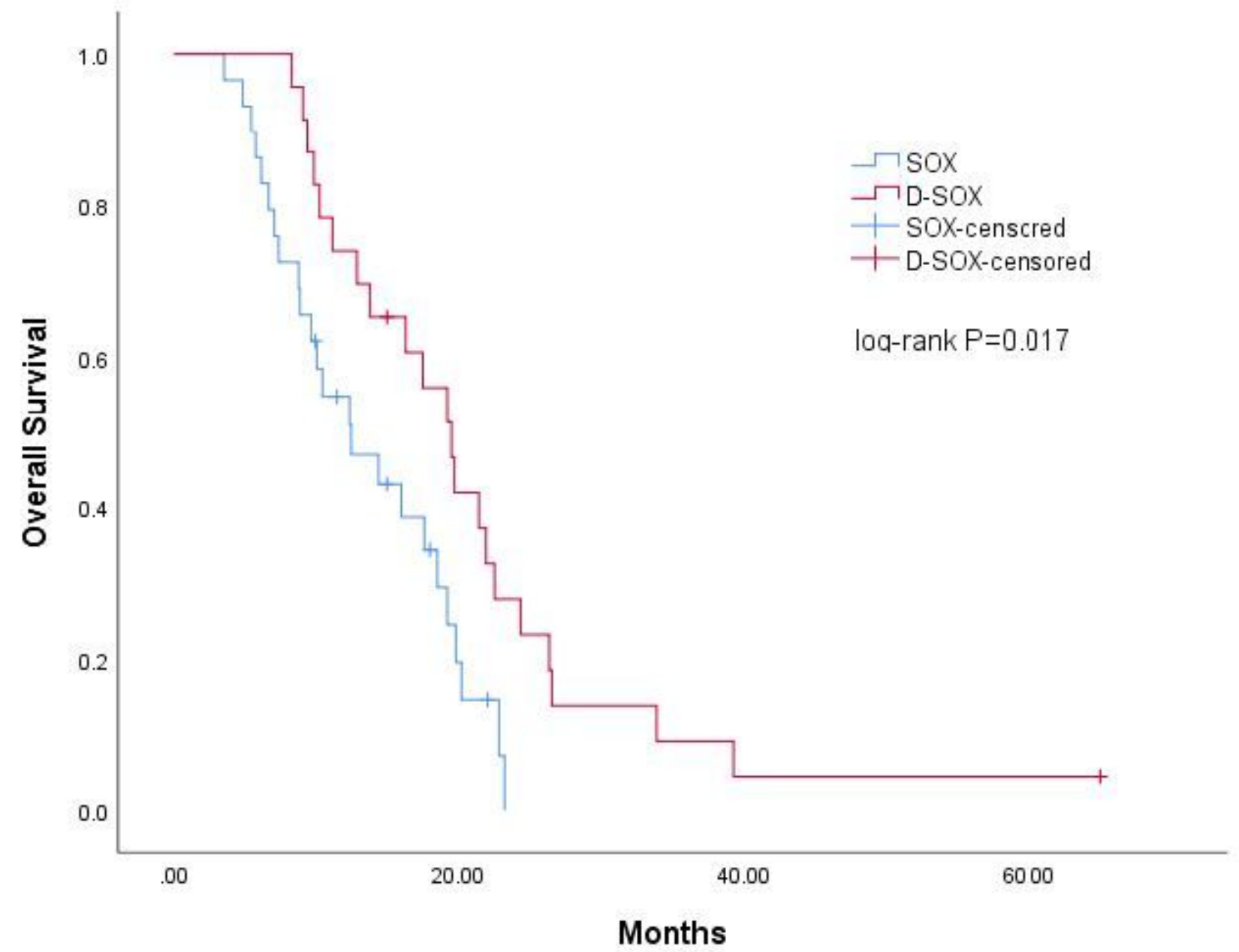

Figure 2

Kaplan-Meier overall survival for D-SOX versus SOX

\section{Supplementary Files}

This is a list of supplementary files associated with this preprint. Click to download.

- TABLE.pdf 